\title{
Emergency Thrombolysis During Cardiac Arrest Due to Pulmonary Thromboembolism: Our Experience Over 6 Years
}

This article was published in the following Dove Press journal: Open Access Emergency Medicine

\author{
David de Paz $\mathbb{D}^{1-4}$ \\ Julio Diez (D)' \\ Fredy Ariza $\mathbb{D}^{2}$ \\ Diego Fernando Scarpetta $(\mathbb{D})^{1-5}$ \\ Jaime A Quintero $\mathbb{D}^{1-3}$ \\ Sandra Milena Carvajal $(\mathbb{D})^{\prime}$ \\ 'Department of Emergency Service, \\ Fundación Valle del Lili, Cali, 760032 , \\ Colombia; ${ }^{2}$ Anesthesia and Perioperative \\ Medicine, Fundación Valle del Lili, Cali \\ 760032, Colombia; ${ }^{3}$ Centro de \\ Investigaciones Clínicas $(\mathrm{ClC})$, Fundación \\ Valle del Lili, Cali 760032, Colombia; \\ ${ }^{4}$ Internal Medicine Residency, \\ Universidad CES, Medellín 05002I, \\ Colombia; ${ }^{5}$ Internal Medicine Residency, \\ Universidad ICESI, Cali, Colombia
}

Correspondence: Sandra Milena Carvajal Department Emergency Service, Fundación Valle del Lili Hospital, Carrera 98 No. 18-49, Cali, 760032, Colombia Email sandra.carvajal@fvl.org.co
Introduction: Cardiac arrest (CA) is one of the leading causes of death worldwide. Among patients with CA, pulmonary embolism (PE) accounts for approximately $10 \%$ of all cases. Objective: To compare the outcomes after cardiopulmonary-cerebral resuscitation (CCPR) with and without thrombolytic therapy (TT) in patients with CA secondary to PE.

Methods: We included patients older than 17 years admitted to our hospital between 2013 and 2017 with a diagnosis of CA with confirmed or highly suspected PE who received CCPR with or without TT. Measures of central tendency were used to depict the data.

Results: The study comprised 16 patients, 8 of whom received CCPR and thrombolysis with alteplase, whereas the remaining patients received CCPR without TT. The most frequent rhythm of $\mathrm{CA}$ in both groups was pulseless electrical activity. Return of spontaneous circulation (ROSC) occurred in $100 \%$ of patients who received TT and in $88 \%$ of nonthrombolysed patients. The mortality rate of patients who received TT and non-thrombolysed patients at 24 hours was $25 \%$ and $50 \%$, respectively. However, at the time of hospital discharge, the mortality was the same in both groups $(62 \%)$. In patients who received TT, mortality was related to sepsis and hemorrhage whereas in non-thrombolysed patients, mortality was due to myocardial dysfunction.

Conclusion: Intra-arrest thrombolysis resulted in a higher likelihood of ROSC and a higher 24-hour survival in adults with CA secondary to acute PE. Overall, the survival at hospital discharge was the same in the two groups.

Keywords: pulmonary embolism, cardiac arrest, return of spontaneous circulation

\section{Introduction}

Cardiac arrest (CA) is one of the leading causes of death worldwide, with an incidence of 28-54 per 100,000 person-years. ${ }^{1}$ Cardiovascular-related causes, especially acute myocardial infarction, are responsible for more than $70 \%$ of all CA. ${ }^{2}$ Pulmonary embolism (PE) accounts for approximately $10 \%$ of cases. ${ }^{3}$ Mortality of patients with CA secondary to PE continues to be high, ranging between $65 \%-88 \%$, despite all the recommended Cardiopulmonary-Cerebral Resuscitation (CCPR) strategies. ${ }^{4,5}$ Between $60 \%$ and $74 \%$ of survivors have a good neurological outcome, with a score of 1-2 on the cerebral performance category (CPC) scale. ${ }^{6,7}$

Advanced CCPR can be supplemented with thrombolysis in patients with CA secondary to $\mathrm{PE}$ as a reperfusion therapy. However, its use in cases of CA remains controversial. ${ }^{8}$ This intervention has been extrapolated from the treatment indicated in patients with high-risk PE defined by hemodynamic instability with arterial 
hypotension or shock. Thrombolytic therapy (TT) has been associated with reduced mortality in such patients. ${ }^{9,10}$ Thrombolysis has been found to be beneficial in return of spontaneous circulation (ROSC) in prospective studies on patients with CA due to all cardiovascular-related causes. ${ }^{11,12}$ Other cohort studies that assessed outcomes with TT in the subgroup of CA secondary to PE found higher rate of ROSC in 24-hour survival and survival to discharge from hospital. ${ }^{6,13-15}$ Conversely, a recent clinical trial found no statistically significant differences in survival nor neurological outcomes with the use of thrombolysis in CA in the subgroup of CA secondary to PE. ${ }^{16}$ International CCPR guidelines consider the use of TT in cases of presumed PE as cause of CA in high-risk patients, being a IIb recommendation, with a C-Limited Data level of evidence. ${ }^{17}$

In Colombia, only case reports with successful outcomes have been published. ${ }^{18,19}$ However, there are no case-control studies to evaluate the use or non-use of TT in CA secondary to PE. Therefore, the purpose of this study was to assess the outcomes of patients with CA secondary to PE who received CCPR with or without TT upon presentation to the emergency department of a tertiary care center/referral university hospital.

\section{Methodology}

\section{Setting and Data Source}

This retrospective observational study was conducted at Fundación Valle del Lili (FVL) University Hospital. The FVL hospital is a non-profit university hospital affiliated with the Icesi University School of Medicine in Cali, Colombia. It serves as a referral facility for critically ill patients from the Southwest region of the country and has an emergency department volume of approximately 70,000 patients per year.

The data for this study were obtained from the FVL institutional registry on cardiac arrest CRECA (Colombian REgistry of Cardiac Arrest). This registry was launched in 2017 to retrospectively collect information of patients with non-traumatic CA. Data collected included demographics, clinical features, resource utilization, and patients' outcomes. The registry searched for data of patients admitted to the emergency department from 2013 to 2017. Information from the registry was complemented by reviewing individual medical charts. This study was approved by our institutional IRB (Register No. 263) and adhered to the standards of the STROBE guidelines
(Strengthening the Reporting of Observational studies in Epidemiology). It was approved by the FVL ethics committee in biomedical research, approved on October 21, 2016. Institutional act No. 21 of October 19, 2016, protocol number 1040. According to resolution 8430 of 1993 of the Colombian Ministry of Health, this study was classified as "no risk"; since it is documentary in nature and the researchers do not assign biological, psychological or social exposures to patients. Therefore, the ethics committee omitted the use of informed consent. All patient data accessed complied with relevant data protection and privacy regulations.

\section{Participants}

Eligibility criteria included patients older than 17 years of both genders from the institutional registry of CA, CRECA, with confirmed or highly suspected PE as the primary cause of the CA and who had received CCPR with or without emergency thrombolysis. Exclusion criteria were non-cardiovascular-related causes of CA, trauma, cancer, or prolonged arrest. Confirmed PE was considered in subjects with documented venous embolism of the pulmonary arteries by computed tomography (CT) angiography. Highly suspected PE was considered in patients with suggestive clinical findings, associated risk factors, and ultrasound criteria.

Case presentation suggestive of PE included symptoms such as dyspnea, pleuritic chest pain, cough, or syncope, and the presence of high-risk factors such as previous venous thromboembolism, immobilization, recent surgery, limb pain, hormonal therapy, or recent childbirth. The ultrasound criteria used were as follows: right ventricle dilation, hypokinesia of the right ventricular wall, flattening of the interventricular septum accompanied by paradoxical motion, and a D-shaped left ventricle.

\section{Variables and Outcomes}

Clinical variables used in the characterization of the study participants included age, gender, medical history, cardiac rhythm, and in- or out-of-hospital CA. To characterize the resuscitation therapy, variables such as medications, dosage, and use or non-use of TT were evaluated. The administration of a thrombolytic agent resulted from a deliberate decision of the emergency physician and the team managing each case. Alteplase, a recombinant tissue plasminogen activator, was used in all patients with TT.

Outcome variables were evaluated in terms of ROSC, 24-hour survival, survival to hospital discharge, 


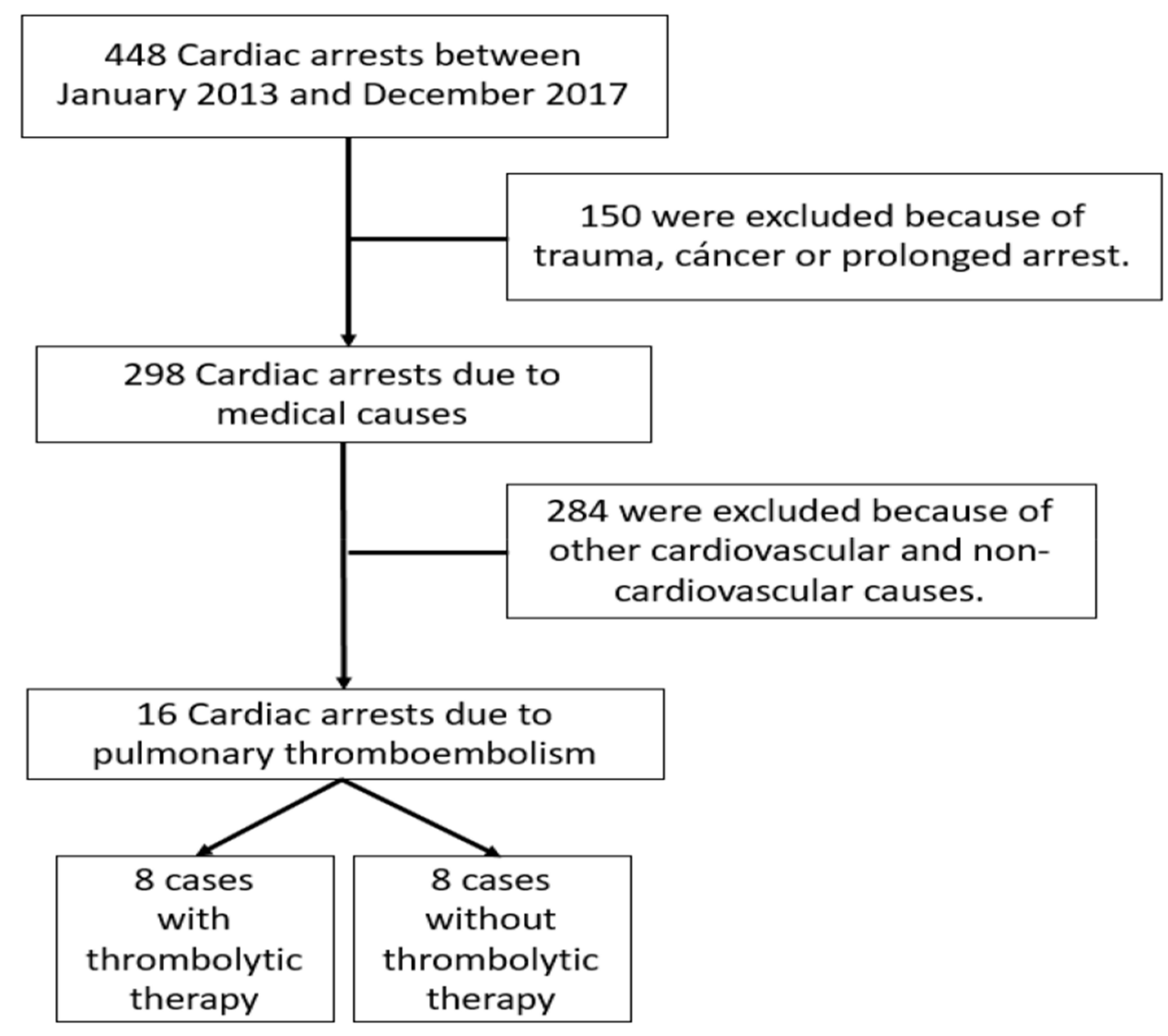

Figure I Code blue registration.

neurological performance, and adverse events derived from the use of a thrombolytic agent. Neurological performance was measured using the CPC disability scale. In essence, the CPC scale classifies patients into five categories: 1) Good cerebral performance $=$ conscious, alert, and able to work; 2) Moderate cerebral disability = conscious, independent with regard to activities of daily living and able to work in a sheltered environment. Patients may present with hemiplegia, seizures, ataxia, dysarthria, dysphasia, or memory or mental changes; 3) Severe cerebral disability $=$ conscious and dependent on others for daily activities; 4) Coma or vegetative state; and 5) Brain death = apnea, areflexia, and EEG silence.

\section{Data Analysis}

Categorical variables were described using frequencies and percentages, and quantitative variables using median and interquartile range (IQR). These descriptions were made by subgroups according to resuscitation decision making, with or without TT. Pearson Chi-2 test and Mann-Whitney $U$-test were used for comparison of categorical and continuous variables, respectively. Analysis was performed using Microsoft $($ ) Excel (Version 16.24, Santa Rosa, California).

\section{Results}

During the study period, 298 patients with CA were treated in the FVL Emergency Department, 16 of whom suffered from acute $\mathrm{PE}$ as the cause of the CA. Eight patients received $\mathrm{CCPR}$ in addition to emergency $\mathrm{TT}$ with alteplase, and the remaining eight received CCPR without thrombolysis (Figure 1).

All patients were older than 50 years, and half of them were male. Among the patients who received TT, the median age was 68 (IQR, 52-80) years, and among the non-thrombolysed patients, the median age was 80 (IQR, $50-85)$ years. Seven subjects $(87.5 \%)$ within the group receiving thrombolysis and six (75\%) from the nonthrombolysed group presented with out-of-hospital CA; of these, four patients within the TT group and one from non-thrombolysed group received CCPR before admission to the hospital. The most frequent $\mathrm{CA}$ rhythm in both groups was pulseless electrical activity in $75 \%$ of patients, followed by asystole. A history of hypertension was 
Table I Clinical Features of Patients According to the Use of Thrombolytic Therapy During Cardiopulmonary Resuscitation

\begin{tabular}{|c|c|c|c|}
\hline Features & TT, n = 8 (\%) & Without TT, n = 8 (\%) & $p$ value \\
\hline Age, median in years (IQR) & $68(52-80)$ & $80(50-85)$ & 0.61 \\
\hline Male gender & $5(62.5)$ & $4(50)$ & 0.61 \\
\hline Confirmatory study & & & 0 \\
\hline CT angiography & $4(50)$ & $4(50)$ & 1.00 \\
\hline FOCUS peri-arrest & $6(75)$ & $5(62.5)$ & 0.59 \\
\hline Initial cardiac arrest rhythm & & & 0 \\
\hline Ventricular fibrillation & I (12.5) & $0(0)$ & 0.30 \\
\hline Pulseless ventricular tachycardia & $0(0)$ & $0(0)$ & 1.00 \\
\hline Pulseless electrical activity & $6(75)$ & $6(75)$ & 1.00 \\
\hline Asystole & I (I2.5) & $2(25)$ & 0.52 \\
\hline Cardiac arrest & & & 0 \\
\hline Out-of-hospital & $7(87.5)$ & $6(75)$ & 0.52 \\
\hline CCPR prior to admission & $4 / 7$ & $1 / 6$ & 0.10 \\
\hline Medication prior to admission & $3 / 7$ & $1 / 6$ & 0.24 \\
\hline \multicolumn{4}{|l|}{ Medical record } \\
\hline Current smoker & I (12.5) & $3(37.5)$ & 0.24 \\
\hline Arterial hypertension & $4(50)$ & $6(75)$ & 0.30 \\
\hline Coronary heart disease & I (I2.5) & $3(37.5)$ & 0.24 \\
\hline Diabetes & $0(0)$ & $3(37.5)$ & 0.06 \\
\hline Heart failure & $0(0)$ & I (I2.5) & 0.30 \\
\hline Lung disease & $2(25)$ & $3(37.5)$ & 0.59 \\
\hline Neurological disease & $0(0)$ & $3(37.5)$ & 0.06 \\
\hline
\end{tabular}

Note: All data, except age, are expressed in terms of frequency (\%).

Abbreviations: CCPR, cerebro-cardio-pulmonary resuscitation; CT, computed tomography; FOCUS, focused cardiac ultrasound; IQR, interquartile range; LV, left ventricle; RV, right ventricle; TT, thrombolytic therapy.

present in $50 \%$ of the TT group and $75 \%$ of patients in the group without TT. Table 1 shows patients' features according to management.

A peri-arrest ultrasound study was performed in $6 / 8$ patients in the group receiving TT and in 5/8 patients in the non-thrombolysed group (Table 2). Signs of right ventricular dysfunction were not found in $1 / 5$ patients. Thrombus in the pulmonary artery was reported in one patient from the TT group. PE was confirmed by computed tomography pulmonary angiogram (CTPA) in 7/16 patients. In $9 / 16$ patients it was confirmed by transthoracic echocardiogram and focused cardiac ultrasound.

ROSC occurred in $100 \%$ of patients undergoing thrombolysis and in $88 \%$ of non-thrombolysed patients; 24 -hour mortality rates were $25 \%$ and $50 \%$, respectively. However, at the time of hospital discharge and at 30-days mortality was equal between TT and non-TT patients. All the survivors showed a good neurological performance according to the CPC scale, except for one patient in the thrombolysis group, who had moderate disability. A surviving patient from the non-thrombolysed group was lost to follow-up after hospital discharge. In the group receiving TT mortality was not related to cardiac dysfunction; two deaths were attributed to sepsis and another to intracranial bleeding. In the non-thrombolysed group, mortality was mainly associated with cardiac dysfunction (Table 3).

Post-interventional adverse events analysis showed two hemorrhagic events occurred in the thrombolysis group; one patient had a minor urinary tract bleed, and the second experienced a fatal intracranial bleeding. In the nonthrombolysed group, no hemorrhagic events were documented.

\section{Discussion}

This study aimed to compare the outcomes of CCPR with and without thrombolysis in patients with CA secondary to $\mathrm{PE}$, identifying a higher ROSC and 24-hour survival in 
Table 2 Findings of Emergency Ultrasound

\begin{tabular}{|l|l|l|l|}
\hline & TT, $\mathbf{n}=\mathbf{6}(\%)$ & Without TT, $\mathbf{n}=\mathbf{5}$ (\%) & p value \\
\hline RV hypokinesia & $6 / 6$ & $4 / 5$ & \\
Ratio RV>LV & $6 / 6$ & $4 / 5$ & 0.59 \\
Septal flattening & $5 / 6$ & $4 / 5$ & \\
Pulmonary artery thrombus & $1 / 6$ & $0 / 5$ & 0.31 \\
FOCUS on the peri-cardiac arrest & $6(75)$ & $5(62.5)$ & \\
\hline
\end{tabular}

Abbreviations: LV, left ventricle; RV, right ventricle; TT, thrombolytic therapy.

those patients who received TT. However, no changes in survival or neurological performance at hospital discharge could be observed.

Our results are similar to those by Bottiger in 2001 and Bozeman in 2006 regarding the increase in ROSC. ${ }^{11,12}$ Bottiger et al conducted a prospective study on $90 \mathrm{CA}$ patients in whom ROSC could not be achieved within the first 15 minutes of CCPR and compared the administration of unfractionated heparin with the use of TT. The group that received TT presented with a higher ROSC rate $(68 \%$ vs. $44 \%, \mathrm{p}=0.026)$, a higher 24 -hour survival rate $(35 \%$ vs. $22 \%, \mathrm{p}=0.171)$, and higher survival to hospital discharge rate $(15 \%$ vs. $8 \%, \mathrm{p}=0.1) .{ }^{11}$ Subsequently, Bozeman et al conducted a prospective study comparing thrombolysis in $50 \mathrm{CA}$ patients who did not respond to
CCPR, against standard CCPR in 113 additional patients. ROSC was higher in the thrombolysed group as compared with control group ( $26 \%$ vs. $12.4 \%, p=0.04)$; however, there were no differences in 24-hour survival or in survival to discharge from hospital. All survivors had a good neurological performance (CPC 1-2). ${ }^{12}$

Small cohort studies from reference centers with similar facilities have found a higher ROSC rate and a higher 24-hour survival rate and survival to hospital discharge rate when administering intra-arrest thrombolysis in patients with PE. ${ }^{6,13-15}$ For example, Lederer et al observed a significant increase in the 24-hour survival rate $(48.1$ vs. $32.9 \%, \mathrm{p}=0.003) .{ }^{15}$ Conversely, our study found no difference in the mortality to hospital discharge rate.

Table 3 Mortality, Neurological Functionality, and Adverse Events Related to the Use of Thrombolysis During Cardiopulmonary Resuscitation

\begin{tabular}{|l|l|l|l|}
\hline Outcomes & TT, $\mathbf{n = 8}$ (\%) & Without TT, $\mathbf{n}=\mathbf{8}$ (\%) & $\mathbf{p}$ value \\
\hline ROSC (\%) & $8(100)$ & $7(87.5)$ & 0.30 \\
\hline Mortality & & & 0 \\
At 24 hours & $2(25)$ & $4(50)$ & 0.30 \\
Upon discharge from the ICU & $5(62.5)$ & $5(62.5)$ & 1.00 \\
To hospital discharge & $5(62.5)$ & $5(62.5)$ & 1.00 \\
At 30 days & $5(62.5)$ & $5 / 7 *$ & 1.00 \\
\hline Causes of death & & & 0 \\
Cardiac & $2 / 5$ & $4 / 5$ & 0.31 \\
Others (e.g., sepsis) & $3 / 5$ & $1 / 5$ & 0.24 \\
\hline Neurological outcome & & & 0 \\
No cerebral disability (CPC I) & $2 / 3$ & $3 / 3$ & 0.59 \\
Moderate cerebral disability (CPC 2) & $1 / 3$ & $0 / 3$ & 0.30 \\
Severe cerebral disability/coma/brain death (CPC 3-5) & $0 / 3$ & $0 / 3$ & 1.00 \\
\hline Hemorrhagic complications & & & 0 \\
Intracerebral hemorrhage & $\mathrm{I}(12.5)$ & $0(0)$ & 0.30 \\
Other hemorrhages & $\mathrm{I}(12.5)$ & $0(0)$ & 0.30 \\
\hline
\end{tabular}

Notes: Data expressed as absolute (\%). *I Lost at follow-up.

Abbreviations: CPC, cerebral performance categories; ICU, intensive care unit; ROSC, return of spontaneous circulation; TT, thrombolytic therapy. 
The TROICA trial published in 2008 by Bottiger et al is perhaps the study that has majorly dismissed the use of intra-arrest thrombolysis. This study included 1050 patients with CA from cardiovascular-related causes; 85 ( $8 \%$ ) of whom presented with documented PE. Further, 525 patients received TT and the remaining patients received a placebo. On comparing the thrombolysed group with the placebo group, they found no statistically significant differences for the primary outcome at the 30day survival mark $(14.7 \%$ vs. $17 \%, \mathrm{p}=0.36)$. In the subgroup of CA secondary to PE, survival was $13.3 \%$ (2/ $15)$ in the thrombolysed group compared with $0 \%(0 / 22)$ in the placebo group $(\mathrm{p}=0.31)$. Our study yields similar results to those in the TROICA study in that no differences were found in neurological outcomes assessed with the CPC scale. ${ }^{16}$

Our cohort study presented one case of intracranial hemorrhage from the subjects who received thrombolysis and none from the non-thrombolysed group. This association of TT with an increased risk of hemorrhage is consistent with other findings in literature, with a low rate of intracranial hemorrhage of less than $5 \%{ }^{12,13,15,16}$

Deaths from the non-thrombolysed group occurred within 24 hours and were mostly due to cardiogenic shock, whereas deaths from patients who received thrombolysis were late and followed non-cardiac complications, mainly septic shock and cerebral hemorrhage. This finding has not been previously observed. This could mean that greater caution must be taken in the post-arrest state in order to prevent such complications and initiate timely management.

In 2019, Karsten Keller et al investigated the use of systemic thrombolysis in hospitalized patients with acute PE in Germany from 2005 to 2015, and analyzed its possible impact on early prognosis. 885806 patients were diagnosed with acute PE. In total, systemic thrombolysis was administered to 36,917 (4.2\%) PE patients.

Among hemodynamically unstable PE patients (excluding those with CCPR), case fatality rates were lower in patients who received thrombolysis compared with those without this reperfusion treatment $(28.6 \%$ vs. $49.9 \%$; $\mathrm{P}<0.001)$. Overall, intracerebral bleeding was more frequent in patients who received systemic thrombolysis compared with those who did not $(1.7 \%$ vs. $0.5 \%$, $\mathrm{P}<0.001) .{ }^{17}$ Our study shows similar outcomes in terms of mortality and complications related to TT; however, it does not meet the statistical power for the generation of hypotheses and analysis of correlation of variables is small.

Focused cardiac ultrasound results in a reliable tool for the diagnosis of PE and as a quick guide for decision making, especially in the unstable patient not tolerating transfer to the CT room. Although its usefulness has not been well established in $\mathrm{CA}$, it should be considered whenever skilled human resource is available and its use does not interfere with standard cardiopulmonary resuscitation. The CTPA was not performed in all patients because of their clinical conditions (high estimated bleeding risk by physician, comorbidities, hemodynamic instability) and the imminent death in the transfer to the CT room.

In our study, the decision for thrombolysis was based on medical criteria. No predictive bleeding scale was used to make this decision. The specialists did not perform the thrombolysis if the patients had contraindications such as active bleeding, predominantly anticoagulation.

Our study has certain limitations, and results should be interpreted in the context of a case series. The small number of cases of CA secondary to PE in this study was 7\%, similar to the prevalence reported for other patients with $\mathrm{CA}$ treated in an emergency department. ${ }^{3,13,16}$ Decision making of emergency physicians regarding the administration of TT to their patients is based on their clinical judgment, and this represents a high risk of selection bias. However, it is possible that many factors not intended in this analysis, such as witnessed CA, elapsed time from out-of-hospital CA, and CCPR duration, were considered.

\section{Conclusion}

Intra-arrest thrombolysis resulted in a greater likelihood of ROSC and a higher 24-hour survival rate in adults with CA secondary to acute PE, although it was not associated with better survival rate to hospital discharge. In our study, thrombolysis was associated with a higher risk of bleeding and sepsis, but a lower likelihood of death from cardiogenic shock as was observed in the non-thrombolysed group during CA. Thus, we recommend that special attention must be taken on strategies that may reduce these complications when thrombolysis is considered. Subsequent studies are needed in this field.

\section{Acknowledgments}

Meetings: CIMER (Congreso Internacional de Medicina de Emergencias y Reanimación CIMER 2019). Emergency Service, Fundación Valle del Lili Cra. 98 \# 18-49, Cali 760032, Cali, Valle del Cauca, Colombia. 


\section{Author Contributions}

All authors contributed to data analysis, drafting or revising the article, have agreed on the journal to which the article will be submitted, gave final approval of the version to be published, and agree to be accountable for all aspects of the work.

\section{Disclosure}

Fredy Ariza report honoraries for scientific consultation from Octapharma, outside the submitted work. The authors report no other potential conflicts of interest for this work.

\section{References}

1. Berdowski J, Berg RA, Tijssen JG, Koster RW. Global incidences of out-of-hospital cardiac arrest and survival rates: systematic review of 67 prospective studies. Resuscitation. 2010;81:1479-1487. doi:10.1016/j.resuscitation.2010.08.006

2. Andersen LW, Holmberg MJ, Berg KM, Donnino MW, Granfeldt A. In-hospital cardiac arrest: a review. JAMA. 2019;321(12):1200-1210. doi:10.1001/jama.2019.1696

3. Comess KA, Derook FA, Russell ML, Tognazzi-evans TA, Beach KW. The incidence of pulmonary embolism in pulseless electrical activity. Am J Med 1998;9343(June):351-356.

4. Kasper W, Konstantinides S, Geibel A, et al. Management strategies and determinants of outcome in acute major pulmonary embolism: results of a multicenter registry. $\mathrm{J} \mathrm{Am}$ Coll Cardiol. 1997;30:1165-1171. doi:10.1016/S0735-1097(97)00319-7

5. Bougouin W, Marijon E, Planquette B, et al. Pulmonary embolism related sudden cardiac arrest admitted alive at hospital: management and outcomes. Resuscitation. 2017;115:135-140. doi:10.1016/j. resuscitation.2017.04.019

6. Er F, Nia AM, Gassanov N, Caglayan E, Erdmann E, Hoppe UC. Impact of rescue-thrombolysis during cardiopulmonary resuscitation in patients with pulmonary embolism. PLoS One. 2009;4(12):2-6. doi:10.1371/journal.pone.0008323

7. Peberdy MA, Kaye W, Ornato JP, et al. Cardiopulmonary resuscitation of adults in the hospital: a report of 14720 cardiac arrests from the national registry of cardiopulmonary resuscitation. Resuscitation. 2003;58:297-308. doi:10.1016/S0300-9572(03)00215-6

8. Jaff MR, McMurtry MS, Archer SL, et al. Management of massive and submassive pulmonary embolism, iliofemoral deep vein thrombosis, and chronic thromboembolic pulmonary hypertension. Circulation. 2011;123 (16):1788-1830. doi:10.1161/CIR.0b013e318214914f
9. Namiranian K, Rathi NK, Banchs J, et al. Does thrombolysis have a place in the cardiopulmonary resuscitation of patients with acute pulmonary embolism? A case of successful thrombolysis during pulmonary embolism induced cardiopulmonary arrest. Crit Care Med. 2016;44(5):e300-e303. doi:10.1097/CCM.0000000000001430

10. Stein PD, Matta F. Thrombolytic therapy in unstable patients with acute pulmonary embolism: saves lives but underused. $\mathrm{Am} \mathrm{J} \mathrm{Med.}$ 2012;125:70-465.

11. Böttiger BW, Bode C, Kern S, et al. Efficacy and safety of thrombolytic therapy after initially unsuccessful cardiopulmonary resuscitation: a prospective clinical trial. Lancet. 2001;357(9268):1583-1585. doi:10.1016/S0140-6736(00)04726-7

12. Bozemana WP, Kleinerb DM, Ferguson KL. Empiric tenecteplase is associated with increased return of spontaneous circulation and short term survival in cardiac arrest patients unresponsive to standard interventions. Resuscitation. 2006;69:399-406. doi:10.1016/j. resuscitation.2005.09.027

13. Kürkciyan I, Meron G, Sterz F, et al. Pulmonary embolism as cause of cardiac arrest: presentation and outcome. Arch Intern Med. 2000;160(10):1529-1535. doi:10.1001/archinte.160.10.1529

14. Janata K, Holzer M, Kürkciyan I, et al. Major bleeding complications in cardiopulmonary resuscitation: the place of thrombolytic therapy in cardiac arrest due to massive pulmonary embolism. Resuscitation. 2003;57(1):49-55. doi:10.1016/S0300-9572(02)00430-6

15. Lederer W, Lichtenberger C, Pechlaner C, Kroesen G, Baubin M. Recombinant tissue plasminogen activator during cardiopulmonary resuscitation in 108 patients with out-of-hospital cardiac arrest. Resuscitation. 2001;50(1):71-76. doi:10.1016/S0300-9572(01)00317-3

16. Böttiger BW, Hans-Richard Arntz MD, Douglas MD, et al.; for the TROICA Trial Investigators and the European Resuscitation Council Study Group. Thrombolysis during resuscitation for out-of-hospital cardiac arrest. $N$ Engl J Med. 2008;359:2651-2662. doi:10.1056/ NEJMoa070570

17. Keller K, Hobohm L, Ebner M, Kresoja K, Konstantinides SV, Lankeit M. Pulmonary circulation trends in thrombolytic treatment and outcomes of acute pulmonary embolism in Germany. Eur Heart J. 2020;41(4):522-529. doi:10.1093/eurheartj/ehz236

18. Wilches N, Rojas MC, Mesa AM, Fajardo LA, Gallego C. Trombólisis intraparo en tromboembolia pulmonar masiva. [Intraarrest thrombolysis in massive pulmonary embolism]. Rev Colomb Cardiol. 2018;25(2):152.e1-152.e6. Spanish.

19. Carvajal SM, Ariza F, De Paz DA, Castaño DA, Early ultrasonographic detection of massive pulmonary embolism. J Emerg Trauma Shock. 2017;10(1):47-48.
The Open Access Emergency Medicine is an international, peerreviewed, open access journal publishing original research, reports, editorials, reviews and commentaries on all aspects of emergency medicine. The manuscript management system is completely online and includes a very quick and fair peer-review system, which is all easy to use. Visit http://www.dovepress.com/testimonials.php to read real quotes from published authors. 